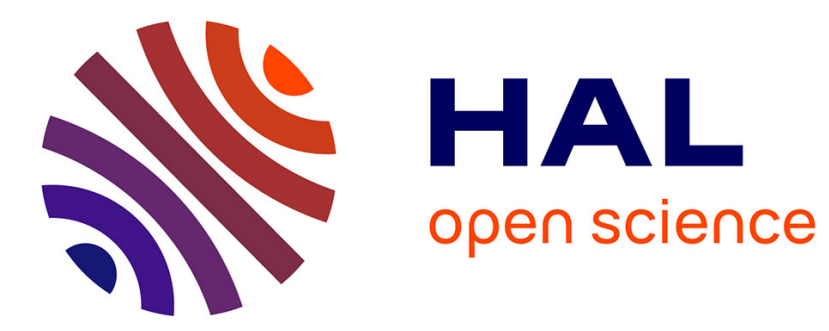

\title{
Existence, number, and stability of limit cycles in weakly dissipative, strongly nonlinear oscillators
}

Michele Bonnin

\section{To cite this version:}

Michele Bonnin. Existence, number, and stability of limit cycles in weakly dissipative, strongly nonlinear oscillators. Nonlinear Dynamics, 2010, 62 (1-2), pp.321-332. 10.1007/s11071-010-9719-1 . hal00589494

\section{HAL Id: hal-00589494 \\ https://hal.science/hal-00589494}

Submitted on 29 Apr 2011

HAL is a multi-disciplinary open access archive for the deposit and dissemination of scientific research documents, whether they are published or not. The documents may come from teaching and research institutions in France or abroad, or from public or private research centers.
L'archive ouverte pluridisciplinaire HAL, est destinée au dépôt et à la diffusion de documents scientifiques de niveau recherche, publiés ou non, émanant des établissements d'enseignement et de recherche français ou étrangers, des laboratoires publics ou privés. 


\title{
Existence, number, and stability of limit cycles in weakly dissipative, strongly nonlinear oscillators
}

\author{
Michele Bonnin
}

Received: date / Accepted: date

\begin{abstract}
Oscillators control many functions of electronic devices, but are subject to uncontrollable perturbations induced by the environment. As a consequence, the influence of perturbations on oscillators is a question of both theoretical and practical importance. In this paper, a method based on Abelian integrals is applied to determine the emergence of limit cycles from centers, in strongly nonlinear oscillators subject to weak dissipative perturbations. It is shown how Abelian integrals can be used to determine which terms of the perturbation are influent. An upper bound to the number of limit cycles is given as a function of the degree of a polynomial perturbation, and the stability of the emerging limit cycles is discussed. Formulas to determine numerically the exact number of limit cycles, their stability, shape and position are given.
\end{abstract}

Keywords Nonlinear oscillators, limit cycles, Abelian integrals, stability.

\section{Introduction}

Oscillators are key components of many electronic devices. For instance, they find application in wireless communication systems for frequency translation of information signals and for channel selection. In digital

This work was partially supported by the Ministero dell'Istruzione, dell'Università e della Ricerca, under the FIRB project no. RBAU01LRKJ. The author thanks the Istituto Superiore Mario Boella and the regional government of Piedmont for financial support.

Michele Bonnin

Department of Electronics, Politecnico di Torino

10129 Turin, Italy

Tel.: +39-011-5644089

Fax: +39-011-5644099

E-mail: michele.bonnin@polito.it electronic systems, they are responsible to provide a clock signal to synchronize operations.

An ideal oscillator would provide a perfect time reference signal. Unfortunately, as any other physical system, electronic devices are corrupted by undesired perturbations such as thermal noise, supply noise, and system-environment interactions. This is often a key performance limiting factor in electronic systems. Characterizing how perturbations affect oscillators is therefore of paramount importance in practical applications, since the characteristics of the local oscillator affect the performance of the entire system.

Periodic oscillations in the time domain correspond to limit cycles in the state space of the oscillator. Thus, a related question of both theoretical and practical relevance concerns the influence of perturbations on the number, position and stability of limit cycles. Since its formulation [1], this question, known as the Hilbert's sixteenth problem, turned out to be one of the most elusive and challenging problems in nonlinear dynamics, with relevant implications into electrical engineering. Arnold suggested a weakened version of Hilbert's sixteenth problem, asking to find a bound to the number of limit cycles which bifurcate from a first order perturbation of a Hamiltonian system $[2,3]$.

An approach to tackle the problem which has enjoyed some popularity in the electrical engineering community is the Melnikov's method [4-8]. Making use of the computable solutions of the unperturbed system, Melnikov's method defines an integral function which measures the distance between two consecutive intersections of the perturbed orbit and a suitable cross section [9]. For autonomous systems the Melnikov's function can be recast as an Abelian integral, and the problem is reduced to counting the zeroes of this integral. Unlike the classical Melnikov's method, Abelian inte- 
grals only rely on the geometrical features of the trajectories, without need to determine the time evolution of the system, which often involves special functions [9].

There is an abundant mathematical literature about Abelian integrals and the number of their zeroes. Usual methods to determine this number are based on PicardFuchs equations, complex analysis, and Chebyshev's property (see $[10,11]$ and references therein). To the best of the author's knowledge, applications of Abelian integrals to circuits and systems are scarce. In this paper the method of Abelian integrals is applied to a strongly nonlinear oscillator under the effect of a polynomial perturbation. A method to determine wether all terms in the perturbation are influent is given. An upper bound to the number of limit cycles (counted with their multiplicities) as a function of the degree of the perturbation is derived, and the stability of the limit cycles is investigated as a function of the coefficients of the polynomial perturbation.

The paper is structured as follows: In section 2 the definition of Abelian integrals, their main properties and their relation to limit cycles are introduced. In section 3 the mathematical model of strongly nonlinear, weakly dissipative oscillators is described. The corresponding Abelian integrals are derived in section 4, where it is shown how they can be used to determine which terms in the perturbation are relevant and which are not. The Picard-Fuchs and the Riccati equations satisfied by these Abelian integrals are derived in section 5, and are used to determine some properties instrumental to find an upper bound to the number of their zeroes. Using these properties, the main theorem concerning the number, positions and stability of the emerging limit cycles is given in section 6 . In section 7 an example is given, and it is shown how the exact number, stability, position and shape of limit cycles can be obtained by numerically solving two simple equations. The theoretical prediction are compared and confirmed with numerical simulations. Section 8 is devoted to conclusions.

\section{Abelian integrals and limit cycles}

Consider the planar autonomous system

$$
\left\{\begin{array}{l}
\dot{x}=\frac{\partial H(x, y)}{\partial y}+\varepsilon f(x, y) \\
\dot{y}=-\frac{\partial H(x, y)}{\partial x}+\varepsilon g(x, y)
\end{array}\right.
$$

where $\varepsilon$ is a small parameter, $H(x, y)$ is a first integral of the unperturbed system $(\varepsilon=0), f(x, y)$ and $g(x, y)$ are polynomials of degree at most $m$. For $\varepsilon=0$ the system is Hamiltonian, so that its equilibrium points are either saddles (unstable) or centers, i.e. neutrally stable surrounded by a continuous family of periodic orbits $\gamma(h)$, which are the level sets $H(x, y)=h$. The question is: How many periodic orbits keep unbroken for small $\varepsilon$ ? To answer this question, consider a cross section $\sigma$ transversal to the level set $\gamma(h)$, and pick an initial condition $h$ on $\sigma$. Let $P(h, \varepsilon)$ be the first return map of the perturbed orbit $\gamma(h, \varepsilon)$ starting from $h$ on $\sigma$. The difference $d(h, \varepsilon)=P(h, \varepsilon)-h$ is called the displacement function (see figure. 1), and the answer to the question above is given by its zeroes.

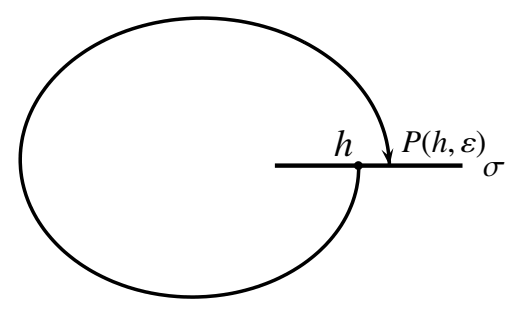

Fig. 1 Construction of the displacement function.

Theorem 1 (Poincaré-Pontryagin [12]) The displacement function is given by

$d(h, \varepsilon)=\varepsilon I(h)+\mathcal{O}\left(\varepsilon^{2}\right)$

as $\varepsilon \rightarrow 0$, where $I(h)$ is the Abelian integral given by

$I(h)=\oint_{\gamma(h)} g(x, y) d x-f(x, y) d y$

Theorem 1 tells that the Abelian integral is a first order measure of the displacement function. If this measure is zero, up to the first perturbative order, the periodic orbit $\gamma(h)$ remains unbroken and a limit cycle of the perturbed system survives to the perturbation, emerging from the center.

The relationships between limit cycles of perturbed Hamiltonian systems and Abelian integrals are summarized in the following theorem:

Theorem $2([\mathbf{1 0 , 1 1 ] )}$ Suppose that $I(h)$ is not identically zero for $h \in(a, b)$, the following statements hold:

- If there exists an $h^{*} \in(a, b)$ such that $I\left(h^{*}\right)=0$ and $I^{\prime}\left(h^{*}\right) \neq 0$, then the perturbed system has a unique hyperbolic limit cycle emerging from $\gamma\left(h^{*}\right)$. The cycle is stable if $I^{\prime}\left(h^{*}\right)<0$, and unstable if $I^{\prime}\left(h^{*}\right)>0$.

- If there exists an $h^{*} \in(a, b)$ such that $I\left(h^{*}\right)=$ $I^{\prime}\left(h^{*}\right)=\ldots=I^{(k-1)}\left(h^{*}\right)=0$ and $I^{(k)}\left(h^{*}\right) \neq 0$, then the perturbed system has at most $k$ limit cycles bifurcating from the same curve $\gamma\left(h^{*}\right)$. 
- The total number of isolated zeroes, counted with their multiplicities, of $I(h)$ for $h \in(a, b)$, is an upper bound to the number of limit cycles, counted with their multiplicities, emerging from the continuous family of periodic orbits $\gamma(h), h \in(a, b)$.

By virtue of this theorem, the problem of finding the number, positions and stability of limit cycles is reduced to counting the zeroes of an Abelian integral, determine their locations and the sign of the derivative at that value of $h$. On the one hand, as it will be shown, these problems are far from being trivial. On the other hand, one can rely on the properties of Abelian integrals, which turn out to be very useful for the solution.

\section{Perturbed nonlinear oscillators}

Planar autonomous systems with cubic nonlinearities and weak dissipative forces have attracted much attention in many areas of applied sciences. Their typical form is

$\ddot{x}+a x+b x^{3}=\varepsilon f(x) \dot{x}$,

and they include Duffing and van der Pol oscillators as special cases. Assuming $\dot{x}=y+\varepsilon F(x)$, where $F(x)$ is a primitive of $f(x)$, computing its derivative and comparing with (4) one obtains the system

$\left\{\begin{array}{l}\dot{x}=y+\varepsilon F(x) \\ \dot{y}=-a x-b x^{3} .\end{array}\right.$

A typical choice for $F(x)$ is a polynomial. In fact, setting apart systems with "dry friction", $F(x)$ is an analytic function and can be represented with any desired accuracy by its Taylor series expansion truncated to a suitable number of terms. Eq. (5) can be rewritten as

$\left\{\begin{array}{l}\dot{x}=y+\varepsilon \sum_{k=1}^{m} c_{k} x^{k} \\ \dot{y}=-a x-b x^{3}\end{array}\right.$

examples of circuits governed by equations of this type are given in $[6,7]$. For $\varepsilon=0$ the system is conservative with Hamiltonian

$H(x, y)=\frac{1}{2} y^{2}+\frac{a}{2} x^{2}+\frac{b}{4} x^{4}$.

Depending on the choice of the parameters $a$ and $b$, the system can exhibit different phase portraits, which go under the names of truncated pendulum case and saddle loop case for $a<0$; global center case, cuspidal loop, and figure-eight loop for $a>0$. This paper focus on the case $a>0, b>0$, known as global center case.
The Hamiltonian (7) is a special case of the one considered in [13], where a sharp bound to the number limit cycles emerging under the effect of a perturbation of low degree is derived. In particular, it is shown that with the linear change of coordinates

$(x, y, t) \mapsto\left(\sqrt{\frac{a}{b}} x, \frac{a}{\sqrt{b}} y, \frac{1}{\sqrt{a}} t\right)$

it is possible to remove the dependence on $a$ and $b$ in the unperturbed system. An upper bound to the number of limit cycles emerging from this simplified Hamiltonian, under the effect of very general polynomial perturbations, has been derived in [14]. In both these papers, however, the problem of the stability of the emerging limit cycles is not tackled, and the methods developed are not suitable to obtain quantitative predictions about limit cycles' position and shape.

In the case under investigation, the scaling (8) transforms system (6) into

$\left\{\begin{array}{l}\dot{x}=y+\varepsilon \frac{\sqrt{b}}{a} \sum_{k=1}^{m} c_{k}\left(\frac{a}{b}\right)^{\frac{k}{2}} x^{k} \\ \dot{y}=-x-x^{3}\end{array}\right.$

simply shifting the dependence on the parameters from the system to the perturbation. One could be tempted to introduce new parameters $\bar{\varepsilon}=\varepsilon \sqrt{b} / a$, and $\bar{c}_{k}=$ $c_{k}(a / b)^{k / 2}$. However, for $a>b$ and $k$ large enough, the coefficients $\bar{c}_{k}$ may eventually grow so large that they cannot be considered as a perturbation anymore.

Returning to (6), for positive values of $a$ and $b$, the system admits a unique equilibrium point at the origin, which is of center type. This equilibrium is surrounded by a continuous family of ovals described by the level curves of $H(x, y)$

$\gamma(h): \frac{1}{2} y^{2}+\frac{a}{2} x^{2}+\frac{b}{4} x^{4}=h, \quad h \in(0,+\infty)$.

Along these curves

$d H(x, y)=y d y+\left(a x+b x^{3}\right) d x=0$.

For $\varepsilon \neq 0$, the origin is still the unique equilibrium point. Assuming $\varepsilon \ll 1$, it is a stable focus for negative values of $c_{1}$, and an unstable focus for positive $c_{1}$. Under the effect of the perturbation, most of the periodic orbits disappear. If the number of those surviving is finite, they become isolated periodic trajectories, e.g. limit cycles, which are said to emerge from the center. 


\section{Abelian integrals for perturbed nonlinear oscillators}

Using equations (3) and (6) the Abelian integral for the perturbed nonlinear oscillator is

$I(h)=-\sum_{k=1}^{m} c_{k} \oint_{\gamma(h)} x^{k} d y$.

One of the goals of this paper is to find an upper bound to the number of zeroes of $I(h)$ as a function of the degree of the perturbation $m$, without solving the integral explicitly. Before to proceed with the analysis, it is convenient to introduce some notation and basic properties of Abelian integrals. It is of use to denote

$I_{n}(h)=\oint_{\gamma(h)} x^{n} y d x$

Integrating by parts one obtains

$I_{n}^{\prime}(h)=\oint_{\gamma(h)} \frac{x^{n}}{y} d x$

where ${ }^{\prime}=\frac{d}{d h}$. An important relation is obtained using Green's formula

$$
\begin{aligned}
\oint_{\gamma(h)} x^{m} y^{n} d y & =m \iint_{H(x, y) \leq h} x^{m-1} y^{n} d x d y \\
& =-\frac{m}{n+1} \oint_{\gamma(h)} x^{m-1} y^{n+1} d x .
\end{aligned}
$$

The following theorems allow to rewrite $I(h)$ in a simpler form and to deduce a number of properties about its zeroes.

Lemma 1 Even powers in the perturbation of (6) do not influence the number of limit cycles, neither their stability.

Proof Using equation (11) the Abelian integral (12) can be rewritten as

$I(h)=\sum_{k=1}^{m} c_{k}\left[a I_{k+1}^{\prime}(h)+b I_{k+3}^{\prime}(h)\right]$.

Applying Green's formula, it is easy to show that $I_{0}(h)$ is the area of the region enclosed by $\gamma(h)$. Denoting by $-\rho$ and $\rho$ the two intersections between $\gamma(h)$ and the $x$-axis yields

$I_{n}^{\prime}(h)=2 \int_{-\rho}^{\rho} \frac{x^{n}}{\sqrt{2 h-a x^{2}-(b / 2) x^{4}}} d x$.

If $n$ is odd, equation (17) is the integral of an odd function evaluated over an interval symmetric with respect to the origin, which is identically null. Thus, for all even values of $k$ in (16) both $I_{k+1}^{\prime}(h)$ and $I_{k+3}^{\prime}(h)$, i.e. those terms in the summation stemming from even powers of the perturbation, are identically null.

As a consequence equation (16) simplifies to

$I(h)=\sum_{k=1}^{l} c_{2 k-1}\left[a I_{2 k}^{\prime}(h)+b I_{2 k+2}^{\prime}(h)\right]$,

where $l=m / 2$ if $m$ is even, $l=(m+1) / 2$ if $m$ is odd. Using equations (14) and (15) and for the same arguments used above one obtains

$I^{\prime}(h)=\sum_{k=1}^{l}(2 k-1) c_{2 k-1} I_{2 k-2}^{\prime}(h)$.

which implies that even powers in the perturbation do not influence the stability of the emerging limit cycles.

Lemma 2 The following recurrence formulas hold

$$
\begin{aligned}
I_{n+4}^{\prime}(h)= & \frac{1}{(n+3) b}\left[4 h(n+1) I_{n}^{\prime}(h)\right. \\
& \left.-2(n+2) a I_{n+2}^{\prime}(h)\right], \\
I_{n}(h)= & \frac{1}{n+3}\left[4 h I_{n}^{\prime}(h)-a I_{n+2}^{\prime}(h)\right] .
\end{aligned}
$$

Proof Multiplying and dividing (14) by $y$, and using (10) one obtains

$I_{n}(h)=2 h I_{n}^{\prime}(h)-a I_{n+2}^{\prime}(h)-\frac{b}{2} I_{n+4}^{\prime}(h)$.

On the other hand, application of Green's formula to equation (14) leads to

$I_{n}(h)=\frac{1}{n+1}\left[a I_{n+2}^{\prime}(h)+b I_{n+4}^{\prime}(h)\right]$.

Eliminating $I_{n}(h)$ from these two equations we get (20), while eliminating $I_{n+4}^{\prime}(h)$ we obtain $(21)$.

Applying equation (20) recursively, any integral $I_{2 n}^{\prime}(h)$ can be brought to depend on the two fundamental integrals $I_{0}^{\prime}(h)$ and $I_{2}^{\prime}(h)$. For instance, the first three integrals are given by

$$
\begin{aligned}
& I_{4}^{\prime}(h)=\frac{4}{3 b}\left[h I_{0}^{\prime}(h)-4 a I_{2}^{\prime}(h)\right] \\
& I_{6}^{\prime}(h)=\frac{4}{15 b^{2}} {\left[-8 a h I_{0}^{\prime}(h)+\left(8 a^{2}+9 b h\right) I_{2}^{\prime}(h)\right] } \\
& I_{8}^{\prime}(h)=\frac{16}{105 b^{3}}\left[h\left(24 a^{2}+25 b h\right) I_{0}^{\prime}(h)\right. \\
&\left.\quad-4 a\left(6 a^{2}+13 b h\right) I_{2}^{\prime}(h)\right] .
\end{aligned}
$$

It follows that both the Abelian integral (18) and its derivative (19) can be written as linear combination of the integrals $I_{0}^{\prime}(h)$ and $I_{2}^{\prime}(h)$. The importance of this result is twofold, in fact both the theoretical and the numerical analysis are greatly simplified, since only two integrals and/or their properties have to be determined. 


\section{Picard-Fuchs and Riccati equations}

A well known fact about Abelian integrals and their ratios is that they satisfy certain types of differential equations, e.g. Picard-Fuchs and Riccati equations. For the sake of completeness, this section is devoted to derive the equations satisfied by the system under investigation. This will provide crucial information about the Abelian integral (18) and its derivative.

Lemma 3 The integrals $I_{0}(h), I_{2}(h)$ and their derivatives satisfy the following Picard-Fuchs equations

$\left(4 b h+a^{2}\right) \frac{d}{d h}\left(\begin{array}{c}I_{0}(h) \\ I_{2}(h)\end{array}\right)=\left(\begin{array}{cc}\frac{3 b h+a^{2}}{h} & \frac{5 a b}{4 h} \\ a & 5 b\end{array}\right)\left(\begin{array}{c}I_{0}(h) \\ I_{2}(h)\end{array}\right)$

$\left(4 b h+a^{2}\right) \frac{d}{d h}\left(\begin{array}{c}I_{0}^{\prime}(h) \\ I_{2}^{\prime}(h)\end{array}\right)=\left(\begin{array}{cc}-b \frac{a b}{4 h} \\ a & b\end{array}\right)\left(\begin{array}{c}I_{0}^{\prime}(h) \\ I_{2}^{\prime}(h)\end{array}\right)$.

The ratio $P(h)=I_{2}^{\prime}(h) / I_{0}^{\prime}(h)$ satisfies the Riccati equation

$\left(4 b h+a^{2}\right) P^{\prime}(h)=a+2 b P(h)-\frac{a b}{4 h} P^{2}(h)$.

Proof Substituting $n=0$ in (20) one obtains

$3 b I_{4}^{\prime}(h)=4 h I_{0}^{\prime}(h)-4 a I_{2}^{\prime}(h)$.

For $n=0$ and $n=2$, equation (21) yields

$$
\left\{\begin{array}{l}
3 I_{0}(h)=4 h I_{0}^{\prime}(h)-a I_{2}^{\prime}(h) \\
5 I_{2}(h)=4 h I_{2}^{\prime}(h)-a I_{4}^{\prime}(h) .
\end{array}\right.
$$

Introducing (30) in the second of (31)

$$
\left\{\begin{aligned}
3 I_{0}(h) & =4 h I_{0}^{\prime}(h)-a I_{2}^{\prime}(h) \\
15 b I_{2}(h) & =-4 a h I_{0}^{\prime}(h)+4\left(3 b h+a^{2}\right) I_{2}^{\prime}(h) .
\end{aligned}\right.
$$

Eliminating $I_{2}^{\prime}(h)$ from these equations, one obtains the first of (27), while eliminating $I_{0}^{\prime}(h)$ the second stems. Taking the derivative of $(32)$, and eliminating $I_{0}^{\prime \prime}(h)$ or $I_{2}^{\prime \prime}(h)$ one obtains $(28)$.

The derivative of $P(h)$ is

$P^{\prime}(h)=\frac{I_{2}^{\prime \prime}(h)}{I_{0}^{\prime}(h)}-\frac{I_{0}^{\prime \prime}(h)}{I_{0}^{\prime}(h)} P(h)$,

multiplying both sides for $\left(4 b h+a^{2}\right)$ and using (28), equation (29) follows.

Theorem 3 The function $P(h)$ is strictly increasing, positive definite and concave in the interval $h \in(0,+\infty)$.
Proof Defining $h=h(t)$, it follows that

$$
\frac{d}{d t} P(h(t))=P^{\prime}(h) \frac{d h}{d t}
$$

and from equation (29)

$$
4 h\left(4 b h+a^{2}\right) P^{\prime}(h)=4 a h+8 b h P(h)-a b P^{2}(h) .
$$

Comparing the two equations

$$
\left\{\begin{aligned}
\frac{d h}{d t} & =4 h\left(4 b h+a^{2}\right) \\
\frac{d P(h)}{d t} & =4 a h+8 b h P(h)-a b P^{2}(h) .
\end{aligned}\right.
$$

For $h \geq 0$, equation (36) has a unique equilibrium point in the origin, which is unstable with one-dimensional center and unstable manifolds. The behavior of solutions can be determined by the method of isoclines [9]. From equation (36)

$\frac{d P(h)}{d h}=\frac{-a b P^{2}(h)+8 b h P(h)+4 a h}{4 h\left(4 b h+a^{2}\right)}$.

The denominator is always positive in the interval $h \in$ $(0,+\infty)$. The roots of the numerator $Q_{ \pm}(h)$, represent invariant sets of $P(h)$, i.e. $P(h)$ cannot intersect these lines, and are given by

$Q_{ \pm}(h)=\frac{4 h}{a} \pm \sqrt{\frac{16 h^{2}}{a^{2}}+\frac{4 h}{b}}$.

These isoclines divide the half plane $\{(h, P(h)): 0<$ $h<+\infty\}$ into three regions, and the vector field (36) is upwards in the middle region, downwards in the upper and lower regions (see figure 2). Routine calculations reveal that

$\left.\frac{d Q_{+}(h)}{d h}\right|_{h=0}=+\infty ;\left.\quad \frac{d P(h)}{d h}\right|_{h=0}=\frac{2}{2 a+b}$.

This implies that $P(h)$ lies in the middle region, and is positive definite, strictly increasing and upper bounded by $Q_{+}(h)$.

To prove that $P(h)$ is concave, compute the derivative of (37)

$$
P^{\prime \prime}(h)=\frac{2 a+4 b P(h)-\left(a b P(h)+12 b h+2 a^{2}\right) P^{\prime}(h)}{8 b h^{2}+2 a^{2} h}
$$

and using (37)

$$
\begin{aligned}
& P^{\prime \prime}(h)=\frac{b(a P(h)-4 h)}{8 h^{2}\left(a^{2}+4 b h\right)^{2}} \times \\
& \quad \times\left[4 a h+2\left(a^{2}+4 b h\right) P(h)+a b P^{2}(h)\right] .
\end{aligned}
$$




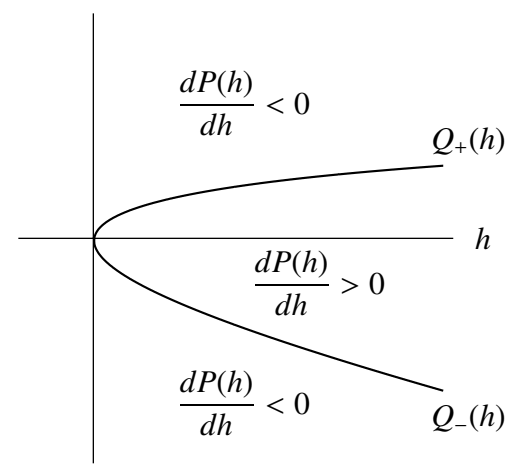

Fig. 2 Behavior of the vector field (36).

Again the denominator is always positive, and $P^{\prime \prime}(h)$ has three roots

$$
\begin{aligned}
R_{0}(h) & =\frac{4 h}{a} \\
R_{ \pm}(h) & =\frac{-\left(a^{2}+4 b h\right) \pm \sqrt{\left(a^{2}+4 b h\right)^{2}-4 a^{2} b h}}{a b} .
\end{aligned}
$$

$R_{0}(h)$, and $R_{ \pm}(h)$ divide the half plane $\{(h, P(h))$ : $0<h<+\infty\}$ into four regions. The second derivative is negative below $R_{-}(h)$ and between $R_{+}(h)$ and $R_{0}(h)$, while it is positive between $R_{-}(h)$ and $R_{+}(h)$, and above $R_{0}(h)$ (see figure 3 ). It is easy to see that $R_{ \pm}(h)$ are negative for all $h>0$, and that

$\left.\frac{d R_{0}(h)}{d h}\right|_{h=0}=\frac{4}{a}$.

Since $2 /(2 a+b)<4 / a$ for all positive values of $a$ and $b$, it follows that $P(h)<R_{0}(h)$, and $P^{\prime \prime}(h)<0$.

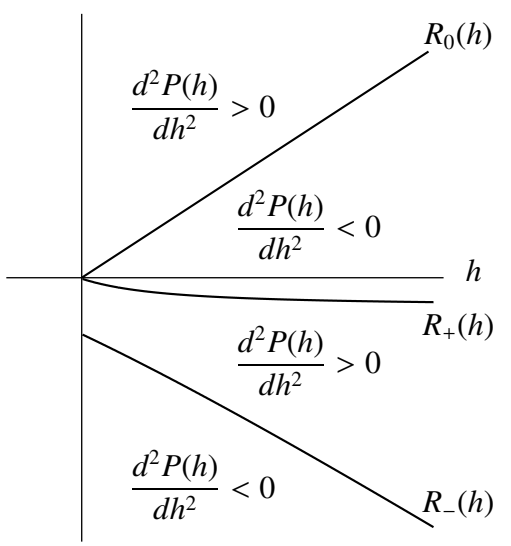

Fig. 3 Sign of the second derivative $P^{\prime \prime}(h)$.

These properties of the function $P(h)$ are instrumental to determine the number of zeroes of $I(h)$ and the sign of its derivative $I^{\prime}(h)$, as it will be shown in the next section.

\section{Number and stability of limit cycles}

This section is devoted to describe the main theorem of the paper, concerning the number, stability and location of limit cycles emerging from the center. A very powerful tool to determine an upper bound to the number of limit cycles emerging in polynomial perturbations of Hamiltonian systems is based on the Chebyshev property $[11,14]$. However, proving that a system enjoys the Chebyschev property requires to introduce rather sophisticated concepts, and often leads to the conclusion that the number of limit cycles is a linear function of the degree of the perturbation $[10,11,14]$. Here, a method developed in [15] will be used; this method is based on simple mathematical concepts and also gives a linear dependence of the number of limit cycles on the degree of the perturbation, despite probably not the strictest one. Before dealing with the theorem, some notations are introduced. In what follows, $p_{r}(h)$, and $q_{s}(h)$ denote polynomials in the variable $h$ of degree $r$ and $s$, respectively; an overbar denotes different polynomials of the same degree.

Eq. (19) can be rewritten in the general form

$I_{n+4}^{\prime}(h)=p_{1}(h) I_{n}^{\prime}(h)+q_{0} I_{n+2}^{\prime}(h)$,

in this way, equations (23)-(26) become

$I_{4}^{\prime}(h)=p_{1}(h) I_{0}^{\prime}(h)+q_{0} I_{2}^{\prime}(h)$

$I_{6}^{\prime}(h)=\bar{p}_{1}(h) I_{0}^{\prime}(h)+q_{1}(h) I_{2}^{\prime}(h)$

$I_{8}^{\prime}(h)=p_{2}(h) I_{0}^{\prime}(h)+\bar{q}_{1}(h) I_{2}^{\prime}(h)$.

By inspecting these equations it is possible to infer a general formula, for $l=2,3, \ldots$

$I_{2 l}^{\prime}(h)=\left\{\begin{array}{l}p_{\frac{l}{2}}(h) I_{0}^{\prime}(h)+q_{\frac{l}{2}-1}(h) I_{2}^{\prime}(h) \text { if } l \text { is even } \\ p_{\frac{l-1}{2}}(h) I_{0}^{\prime}(h)+q_{\frac{l-1}{2}}(h) I_{2}^{\prime}(h) \text { if } l \text { is odd }\end{array}\right.$

Equations (18) and (19) become

$$
\begin{aligned}
I(h) & =p_{r}(h) I_{0}^{\prime}(h)+q_{s}(h) I_{2}^{\prime}(h) \\
I^{\prime}(h) & =p_{r-1}(h) I_{0}^{\prime}(h)+q_{s-1}(h) I_{2}^{\prime}(h)
\end{aligned}
$$

where, for $l=2,3, \ldots$

$(r, s)=\left\{\begin{array}{cl}\left(\frac{l+1}{2}, \frac{l-1}{2}\right) & \text { if } l \text { is odd } \\ \left(\frac{l}{2}, \frac{l}{2}\right) & \text { if } l \text { is even }\end{array}\right.$

Theorem 4 An upper bound to the number of limit cycles, counted with their multiplicities, is:

- Zero, for $m \leq 2$. 
- One, for $2<m \leq 4$, provided $p_{0} / q_{0}<0$, and zero otherwise. The cycle is stable if $q_{0}<0$, unstable otherwise.

- Two, for $4<m \leq 6$; The inner cycle is stable and the outer unstable for $q_{0}<0$. For $q_{0}>0$ the stability is reversed.

- For $m>6$, an upper bound to the number of limit cycle is given by:

$$
j=\left\{\begin{array}{c}
\frac{3 l+1}{2} \text { if } l \text { is odd } \\
\frac{3 l}{2} \text { if } l \text { is even. }
\end{array}\right.
$$

Proof The proof is based on the facts that between two consecutive zeroes of $I(h)$ there is always at least one zero of $I^{\prime}(h)$, and that $h=0$ is a double zero of $I(h)$, i.e. $I(0)=I^{\prime}(0)=0$.

For $m \leq 2$, i.e. $l=1$, equations (50) and (51) become

$$
\begin{aligned}
I(h) & =p_{1}(h) I_{0}^{\prime}(h)+q_{0} I_{2}^{\prime}(h) \\
I^{\prime}(h) & =\bar{q}_{0} I_{0}^{\prime}(h) .
\end{aligned}
$$

It is trivial to observe that for $h \in(0,+\infty), I^{\prime}(h)$ is always different from zero, so that $I(h)$ is either strictly increasing or strictly decreasing. In both cases it cannot have zeroes for $h \in(0,+\infty)$.

For $2<m \leq 4$, i.e. $l=2$, the following equations hold

$$
\begin{aligned}
I(h) & =p_{1}(h) I_{0}^{\prime}(h)+q_{1}(h) I_{2}^{\prime}(h) \\
I^{\prime}(h) & =p_{0} I_{0}^{\prime}(h)+q_{0} I_{2}^{\prime}(h) .
\end{aligned}
$$

Equation (56) can be rewritten as

$I^{\prime}(h)=I_{0}^{\prime}(h)\left(p_{0}+q_{0} P(h)\right)$.

If $p_{0} / q_{0}<0, I^{\prime}(h)$ has a unique zero at $\bar{h} \in(0,+\infty)$, and as a consequence $I(h)$ has a unique zero at $h^{*}>\bar{h}$. Since $P(h)$ is a strictly increasing function, $P\left(h^{*}\right)>$ $P(\bar{h})$, see figure 4 . Then $I^{\prime}\left(h^{*}\right)>I^{\prime}(\bar{h})=0$ if $q_{0}>$ 0 , and the cycle is unstable. Conversely, for $q_{0}<0$, $I^{\prime}\left(h^{*}\right)<I^{\prime}(\bar{h})=0$ and the cycle is stable.

For $4<m \leq 6$, i.e. $l=3$, equations (50), (51) yield

$$
\begin{aligned}
I(h) & =p_{2}(h) I_{0}^{\prime}(h)+q_{1}(h) I_{2}^{\prime}(h) \\
I^{\prime}(h) & =p_{1}(h) I_{0}^{\prime}(h)+q_{0} I_{2}^{\prime}(h) .
\end{aligned}
$$

Rewriting (59) as

$I^{\prime}(h)=I_{0}^{\prime}(h)\left(p_{1}(h)+q_{0} P(h)\right)$,

it is easy to see that $I^{\prime}(h)$ can have at most two zeroes in $(0,+\infty)$, given by the intersections between the straight line $-p_{1}(h) / q_{0}$ and $P(h)$, at $\bar{h}_{1}, \bar{h}_{2}$. Assuming $\bar{h}_{1}<\bar{h}_{2}$,

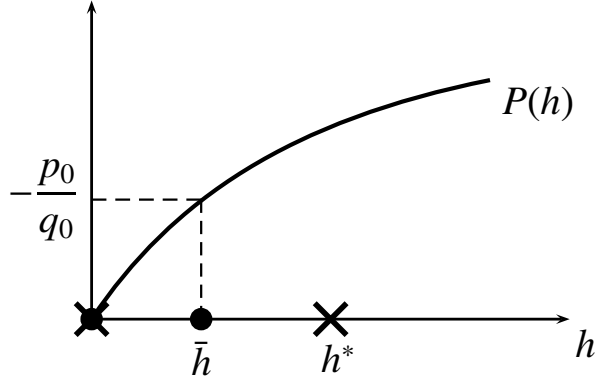

Fig. 4 Zeros of the Abelian integral $I(h)$ (crosses) and its derivative $I^{\prime}(h)$ (dots), given by equations (55) and (56). The position of $h^{*}$ is only indicative.

$I(h)$ can have at most two zeroes at $h_{1}^{*}, h_{2}^{*}$, with $\bar{h}_{1}<$ $h_{1}^{*}<\bar{h}_{2}<h_{2}^{*}$. Since $P(h)$ is a concave function, it follows that

$\frac{p_{1}\left(h_{2}^{*}\right)}{q_{0}}+P\left(h_{2}^{*}\right)<0<\frac{p_{1}\left(h_{1}^{*}\right)}{q_{0}}+P\left(h_{1}^{*}\right)$.

The situation is summarized in figure 5 .

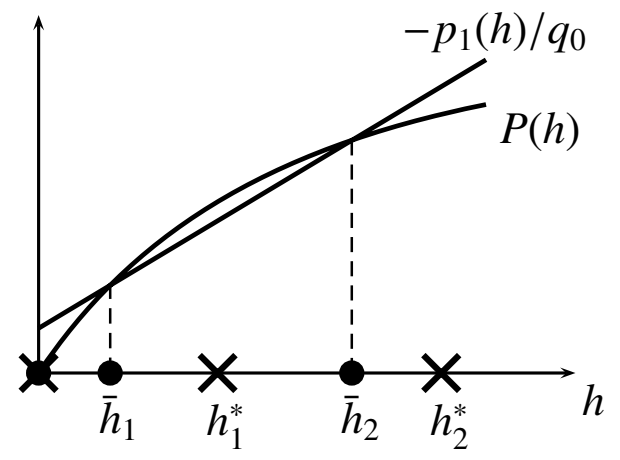

Fig. 5 Zeros of the Abelian integral $I(h)$ (crosses) and its derivative $I^{\prime}(h)$ (dots) given by equations (58) and (59). The position of $h_{1}^{*}$ and $h_{2}^{*}$ is indicative.

If $q_{0}>0$, then $p_{1}\left(h_{2}^{*}\right)+q_{0} P\left(h_{2}^{*}\right)<0<p_{1}\left(h_{1}^{*}\right)+$ $q_{0} P\left(h_{1}^{*}\right)$ and $I^{\prime}\left(h_{2}^{*}\right)<0<I^{\prime}\left(h_{1}^{*}\right)$. This implies that the inner cycle emerging from $\gamma\left(h_{1}^{*}\right)$ is unstable, while the outer emerging from $\gamma\left(h_{2}^{*}\right)$ is stable. By the same arguments it is easy to see that the stability is reversed for $q_{0}<0$.

For $m>6$, i.e. $l \geq 4$, consider (50) and (51). Eliminating $I_{0}^{\prime}(h)$ one gets

$p_{r}(h) I^{\prime}(h)=p_{r-1}(h) I(h)+M(h)$

where

$M(h)=\left[p_{r}(h) q_{s-1}(h)-p_{r-1}(h) q_{s}(h)\right] I_{2}^{\prime}(h)$.

Let $h_{1}, h_{2}$, with $h_{1}<h_{2}$, be two consecutive simple zeroes of $I(h)$, i.e. $I\left(h_{i}\right)=0, I^{\prime}\left(h_{i}\right) \neq 0, i=1,2$. Then $I^{\prime}\left(h_{1}\right) I^{\prime}\left(h_{2}\right)<0$. Equation (62) implies that either there exists $\bar{h} \in\left(h_{1}, h_{2}\right)$ such that $p_{r}(\bar{h})=0$, 
or $M\left(h_{1}\right) M\left(h_{2}\right)<0$, which implies the existence of $h^{*} \in\left(h_{1}, h_{2}\right)$ such that $M\left(h^{*}\right)=0$. It follows that between two simple zeroes of $I(h)$, there exist at least either a zero of $p_{r}(h)$, or of $M(h)$, or both.

Conversely, let $\bar{h}$ be a zero of $I(h)$ with multiplicity $n$. Then it is a zero of both $I^{\prime}(h)$ and $M(h)$ with multiplicity $n-1$.

In both cases, denoting by $j$ the number of zeros of $I(h)$, by $u$ those of $p_{r}(h)$ and by $v$ those of $M(h)$ one has

$j \leq u+v+1$.

Clearly $u=r, v=r+s-1$, and the thesis follows.

\section{Application}

As an example consider the system

$\left\{\begin{array}{l}\dot{x}=y+\sum_{k=1}^{6} c_{k} x^{k} \\ \dot{y}=-a x-b x^{3} .\end{array}\right.$

The corresponding Abelian integral and its derivative are

$I(h)=\sum_{k=1}^{3} c_{2 k-1}\left[a I_{2 k}^{\prime}(h)+b I_{2 k+2}^{\prime}(h)\right]$

$I^{\prime}(h)=\sum_{k=1}^{3}(2 k-1) c_{2 k-1} I_{2 k-2}^{\prime}(h)$.

Using the recurrence formulas (20) and (21) one obtains

$$
\begin{aligned}
I(h) & =\left(\alpha h^{2}+\beta h\right) I_{0}^{\prime}(h)+(\gamma h+\delta) I_{2}^{\prime}(h) \\
I^{\prime}(h) & =\left(c_{1}+\frac{20 c_{5}}{3 b} h\right) I_{0}^{\prime}(h)+\left(3 c_{3}-\frac{20 a c_{5}}{3 b}\right) I_{2}^{\prime}(h) .
\end{aligned}
$$

where

$$
\begin{aligned}
& \alpha=\frac{80 c_{5}}{21 b} \quad \beta=\frac{4 c_{1}}{3}-\frac{4 a c_{3}}{5 b}+\frac{32 a^{2} c_{5}}{21 b^{2}} \\
& \gamma=\frac{12 c_{3}}{5}-\frac{116 a c_{5}}{21 b} \delta=-\frac{a c_{1}}{3}+\frac{4 a^{2} c_{3}}{5 b}-\frac{32 a^{3} c_{5}}{21 b^{2}} .
\end{aligned}
$$

Limit cycles emerges from trajectories associated to values of $h$ such that

$P(h)=-\frac{\alpha h^{2}+\beta h}{\gamma h+\delta}$,

while the zeroes of $I^{\prime}(h)$ are obtained solving

$P(h)=\frac{20 c_{5} h+3 b c_{1}}{20 a c_{5}-9 b c_{3}}$.

The solutions to equation (71) are given by the intersections between a straight line and the strictly increasing, concave function $P(h)$. Restricting the analysis to positive values of $c_{5}$ (negative values does not pose any particular problem and can be treated in analogous way), one finds that:

$-20 a c_{5}-9 b c_{3}<0$ implies $q_{0}>0$, and that the straight line has negative slope. For $c_{1}>0$ the origin is an unstable equilibrium point, and the straight line intercepts the $y$-axe at a negative value, thus there are not limit cycle. For $c_{1}<0$, the origin is stable and the straight line intercepts the $y$-axe at a positive value, this condition is sufficient to have at most one limit cycle. Since $P\left(h^{*}\right)>-p_{1}\left(h^{*}\right) / q_{0}$, $I^{\prime}\left(h^{*}\right)>0$ and the cycle is unstable.

$-20 a c_{5}-9 b c_{3}>0$ implies $q_{0}<0$ and that the straight line has positive slope. For $c_{1}<0$ the origin is stable and the straight line intersects the $y$-axe at a negative point, but this condition alone does not guarantee that there exists one intersection. If such intersection exists, $P\left(h^{*}\right)<-p_{1}\left(h^{*}\right) / q_{0}, I^{\prime}\left(h^{*}\right)>0$ and the cycle is unstable. Conversely, for $c_{1}>0$ the origin is unstable, and the straight line intersects the $y$-axe at a positive point, this condition is necessary, but not sufficient to have two limit cycles. If there is only one limit cycle, one has $P\left(h^{*}\right)>-p_{1}\left(h^{*}\right) / q_{0}$, that is $I^{\prime}\left(h^{*}\right)<0$ and the cycle is stable. If there are two intersections, by virtue of theorem 4 the inner is stable and the outer unstable.

In the general case, to determine the exact values $h^{*}$ at which limit cycles emerge from $\gamma(h)$, one should solve equation (50). The stability of the emerging limit cycles can be determined by looking at the sign of equation (51) at $h=h^{*}$. This can be done numerically using the formulas for $I_{0}^{\prime}(h), I_{2}^{\prime}(h)$ and the complete elliptic integrals given in the appendix. In order to confirm the theoretical results, such numerical calculations have been performed for the example above with $a=2, b=3$, and different values of the parameters $c_{1}, c_{3}$ and $c_{5}$. The results are shown in figures $6-9$, in the upper part it is shown the behavior of $P(h)$ (solid line), the right hand side of (70) (dashed line) and of (71) (dotted line); in the lower part it is shown the behavior of (65) obtained through numerical simulations. Figure (10) shows the comparison between the shape and position of the stable limit cycle predicted for $c_{1}=1, c_{3}=-4, c_{5}=2.5$ and the numerically obtained limit cycle.

\section{Conclusions}

Determine the existence, number, position and stability of limit cycles in nonlinear oscillators is a problem of paramount importance in many areas of applied sciences and engineering. An analytical tool which gives 

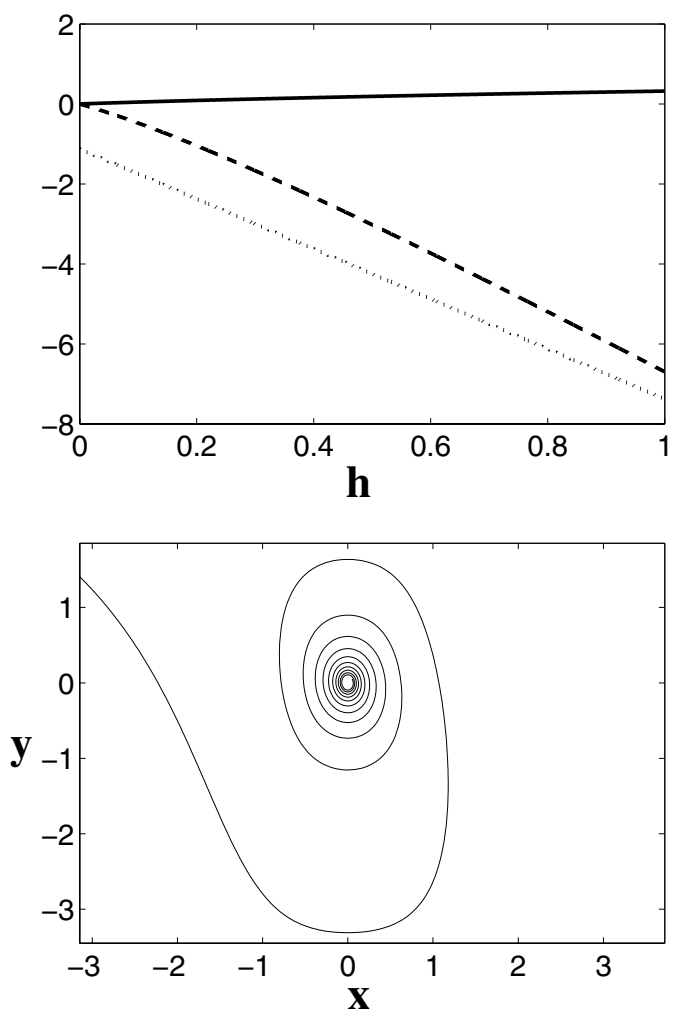

Fig. 6 Upper: Plots of $P(h)$ (solid line), and the left hand side of (70) (dashed line) and (71) (dotted line). Since (70) has no real roots for $h \in(0,+\infty)$, limit cycles cannot exist. Lower: The result is confirmed by numerical simulations of (65), the origin is an unstable equilibrium point, and there are not limit cycles. Values of the parameters: $c_{1}=1, c_{3}=4, c_{5}=2.5$.

an a priori knowledge about the issues above, can help designers and save time with numerical simulations.

In this paper, the method of Abelian integrals has been applied to investigate the emergence of limit cycles in strongly nonlinear oscillators with weak dissipative perturbations. These systems can model, for instance, van der Pol and Duffing oscillators in the weakly dissipative limit. Under the effect of a perturbation, Hamiltonian centers are destroyed, and the emerging limit cycles are related to the zeroes of Abelian integrals. Thus, the research of the former is reduced to finding the latter. The sign of the derivative of the Abelian integrals determine the stability of the limit cycle.

To solve the problem, one can take advantage of the many properties enjoyed by Abelian integrals, e.g. they satisfy recurrence relations, Picard-Fuchs and Riccati equations. Using these properties, both the Abelian integral and its derivative can be rewritten, for polynomial perturbations of any degree, as a linear combination of two basis integral functions, only.

By looking at these integrals, it is possible to determine wether all terms in the perturbation have influence on the emergence of limit cycles and their stability. Re-
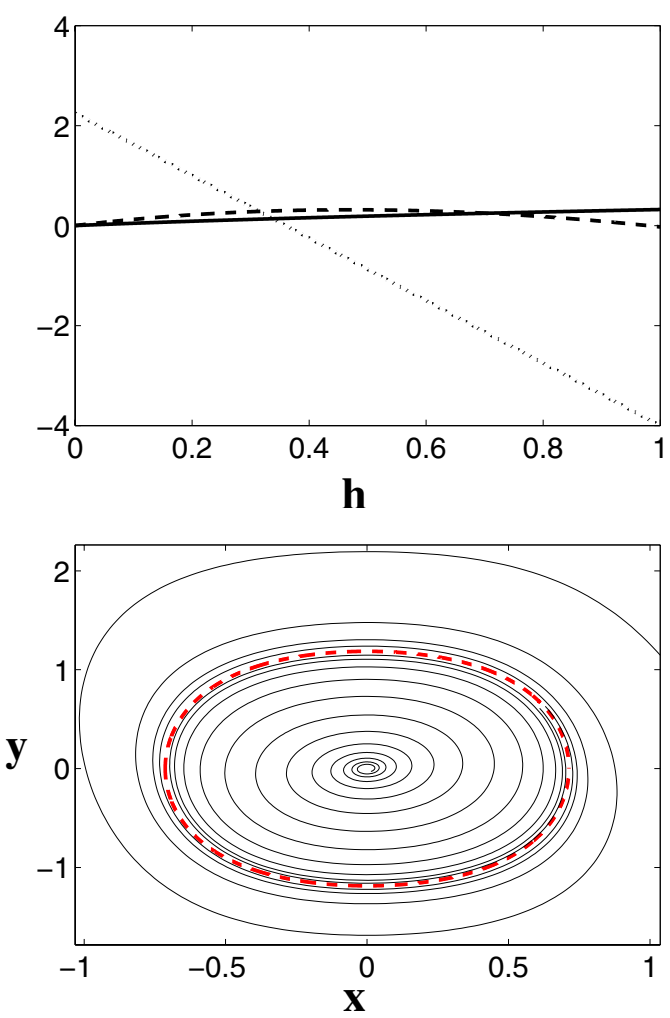

Fig. 7 Upper: Equation (70) has one real roots $h^{*}$ and thus there is one limit cycle. By equation (71) the cycle is unstable. Lower: Numerical simulations confirm the existence of an unstable limit cycle. The limit cycle emerges from the level curve $\gamma\left(h^{*}\right)$ with $h^{*}=0.7034$. The shape of this cycle has been plotted using (10) (thick dashed line). Values of the parameters: $c_{1}=-2, c_{3}=4$, $c_{5}=2.5$.

sorting to basic mathematical concepts, it is possible to find an upper bound to the number of zeroes of the Abelian integral. This upper bound is a linear function of the degree of the perturbation. The stability of the emerging limit cycles can be determined as a function of the coefficients of the perturbations. Although the relation between the sign of the derivative of Abelian integrals and stability is well known, seldom has this possibility been explored.

As shown in the example, the developed technique is suitable to be numerically exploited to determine the exact number, stability, position and shape of the emerging limit cycles. The theoretical results are confirmed by numerical simulations.

\section{Appendix}

This appendix is devoted to the solutions of the integrals $I_{0}^{\prime}(h)$ and $I_{2}^{\prime}(h)$, and to prove that $P(h)$ goes to zero as $h$ goes to zero. By the definition (17) one has

$I_{0}^{\prime}(h)=4 \sqrt{2} \int_{0}^{\rho} \frac{d x}{\sqrt{4 h-2 a x^{2}-b x^{4}}}$ 

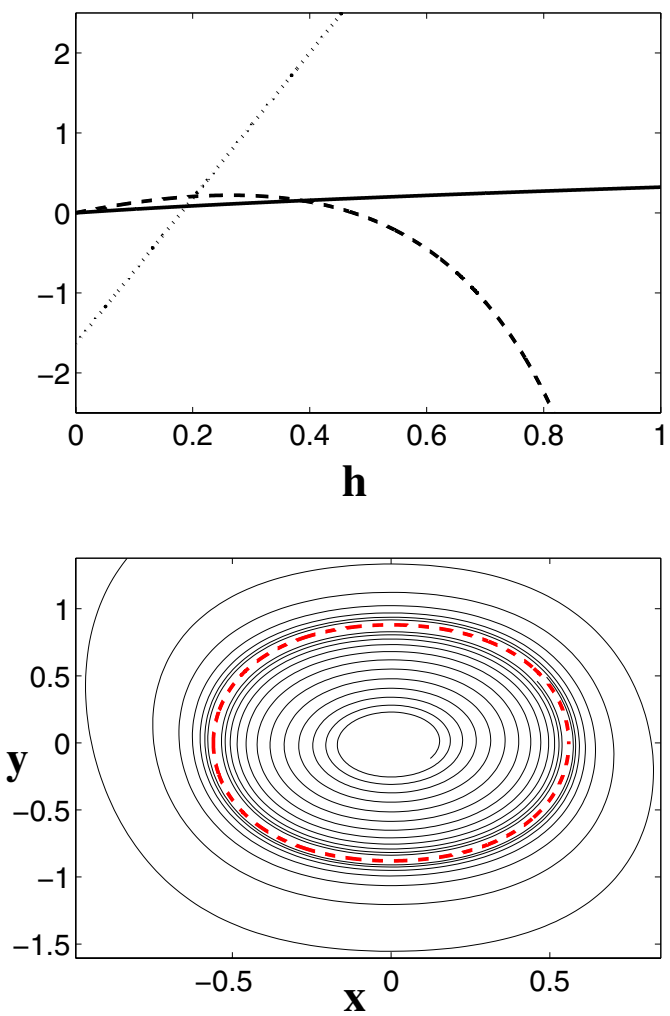

Fig. 8 Upper: Equation (70) has one real roots $h^{*}$ and thus there is one limit cycle. By equation (71) the cycle is unstable. Lower: Numerical simulations confirm the existence of an unstable limit cycle. The limit cycle emerges from the level curve $\gamma\left(h^{*}\right)$, with $h^{*}=4.7453$. The shape of this cycle has been plotted using (10) (thick dashed line). Values of the parameters: $c_{1}=-1, c_{3}=3.5$, $c_{5}=2.5$.

where

$\rho=\sqrt{\frac{1}{b}\left(-a+\sqrt{a^{2}+4 b h}\right)}$.

Introducing

$\sigma=\sqrt{\frac{1}{b}\left(a+\sqrt{a^{2}+4 b h}\right)}$,

one obtains

$I_{0}^{\prime}(h)=4 \sqrt{\frac{2}{b}} \int_{0}^{\rho} \frac{d x}{\sqrt{\left(\rho^{2}-x^{2}\right)\left(\sigma^{2}+x^{2}\right)}}$,

which can be rewritten as

$I_{0}^{\prime}(h)=\frac{4}{\rho \sigma} \sqrt{\frac{2}{b}} \int_{0}^{\rho}\left[\left(1-\frac{x^{2}}{\rho^{2}}\right)\left(1+\frac{x^{2}}{\sigma^{2}}\right)\right]^{-1 / 2} d x$.

Substituting $y=x / \rho$ and $m=-\rho^{2} / \sigma^{2}$ yields

$I_{0}^{\prime}(h)=\frac{4}{\sigma} \sqrt{\frac{2}{b}} \int_{0}^{1} \frac{d y}{\sqrt{\left(1-y^{2}\right)\left(1-m y^{2}\right)}}=\frac{4}{\sigma} \sqrt{\frac{2}{b}} K(m)$.

where $K(m)$ is the complete elliptic integral of the first kind. The properties of complete elliptic integrals, even
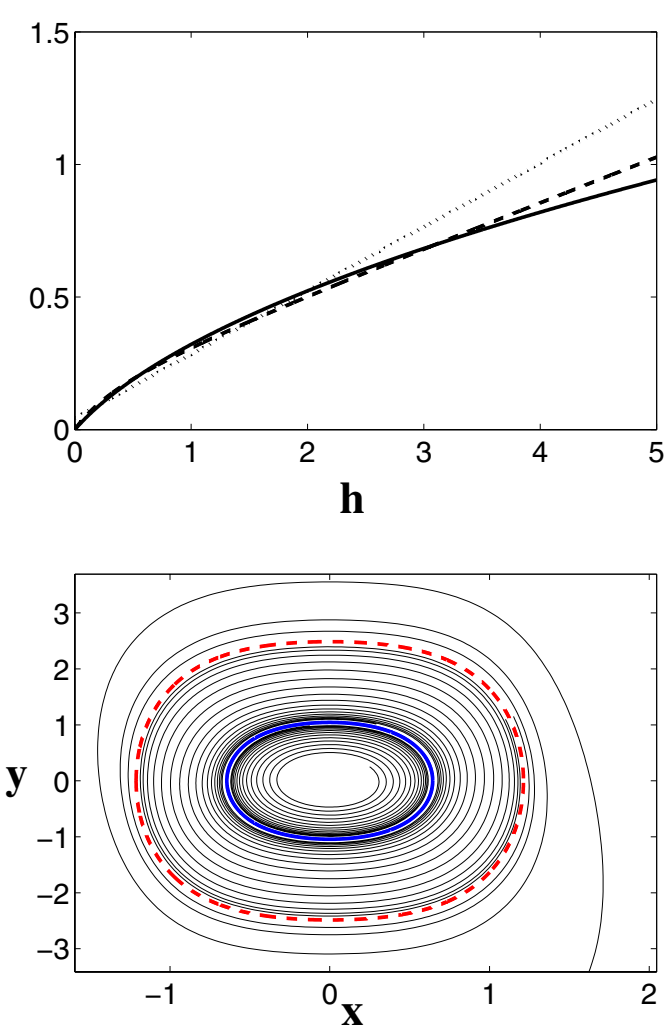

Fig. 9 Upper: Equation (70) has two real roots and thus there are two limit cycles. By equation (71) the inner is stable and the outer is unstable. Lower: Numerical simulations show a stable (thick solid line) and an unstable (thick dashed line) limit cycle. The limit cycles emerge from the level curves $\gamma\left(h_{1}^{*}\right)$, and $\gamma\left(h_{2}^{*}\right)$, plotted using (10) with $h_{1}^{*}=0.5446$, and $h_{2}^{*}=3.0893$. Values of the parameters: $c_{1}=1, c_{3}=-4, c_{5}=2.5$.

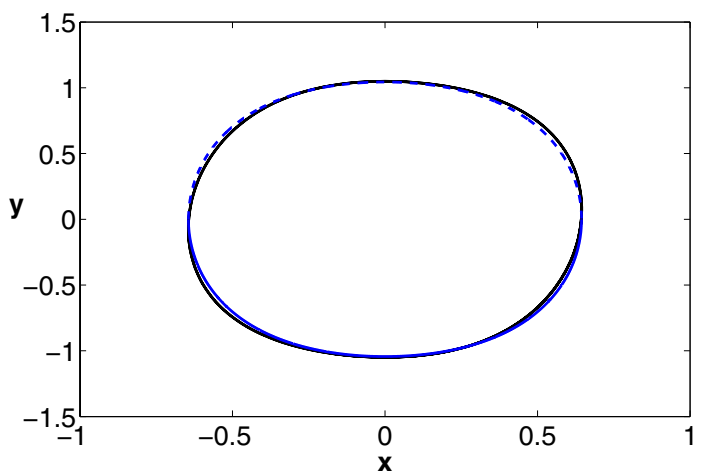

Fig. 10 Comparison of the shape and position of the predicted stable limit cycle (dashed line) and the numerically obtained one (solid line) for $c_{1}=1, c_{3}=-4, c_{5}=2.5$. To emphasize the difference, the perturbation is rather strong, $\varepsilon=0.5$.

for negative values of the parameter $m$ as in this case, are well known and efficient numerical algorithms for their calculation are available [16].

For the integral $I_{2}^{\prime}(h)$, from (17) one has

$I_{2}^{\prime}(h)=4 \sqrt{2} \int_{0}^{\rho} \frac{x^{2}}{\sqrt{4 h-2 a x^{2}-b x^{4}}} d x$. 
Working as before leads to

$$
\begin{aligned}
I_{2}^{\prime}(h)= & 4 \sqrt{\frac{2}{b}}\left[\int_{0}^{\rho} \frac{\sqrt{\sigma^{2}+x^{2}}}{\sqrt{\rho^{2}-x^{2}}} d x\right. \\
& \left.-\sigma^{2} \int_{0}^{\rho} \frac{d x}{\sqrt{\left(\rho^{2}-x^{2}\right)\left(\sigma^{2}+x^{2}\right)}}\right]
\end{aligned}
$$

The second integral on the right hand side has already been solved. Using the same substitution introduced above, the first one becomes

$\int_{0}^{\rho} \frac{\sqrt{\sigma^{2}+x^{2}}}{\sqrt{\rho^{2}-x^{2}}} d x=\sigma \int_{0}^{1} \frac{\sqrt{1-m y^{2}}}{\sqrt{1-y^{2}}} d y=\sigma E(m)$

where $E(m)$ is the complete elliptic integral of the second kind. Putting everything together

$I_{2}^{\prime}(h)=4 \sigma \sqrt{\frac{2}{b}}[E(m)-K(m)]$.

Computing the ratio one gets

$P(h)=\frac{I_{2}^{\prime}(h)}{I_{0}^{\prime}(h)}=\sigma^{2}\left[\frac{E(m)}{K(m)}-1\right]$.

For $|m|<1$, the complete elliptic integrals admit the following infinite series expansions [16],

$K(m)=\frac{\pi}{2}\left\{1+\sum_{n=1}^{+\infty}\left[\frac{(2 n-1) ! !}{(2 n) ! !}\right]^{2} m^{n}\right\}$
$E(m)=\frac{\pi}{2}\left\{1-\sum_{n=1}^{+\infty}\left[\frac{(2 n-1) ! !}{(2 n) ! !}\right]^{2} \frac{m^{n}}{2 n-1}\right\}$.

Taking into account that, by the definition of $m, \rho$ and $\sigma, h \rightarrow 0$ implies $m \rightarrow 0$, it follows that

$\lim _{h \rightarrow 0} \frac{E(m)}{K(m)}=1$

and

$\lim _{h \rightarrow 0} P(h)=0$.

\section{References}

1. D. Hilbert, "Mathematical problems," Bulletin of the American Mathematical Society, vol. 8, pp. 407-436, 1902, (M. Newton translation).

2. V. I. Arnold, "Loss of stability of self-oscillations close to resonance and versal deformations of equivariant vector fields," Functional Analysis Applications, vol. 11, pp. 85-92, 1977.

3. V. I. Arnold, "en Problems," Advances Soviet Mathematics, vol. 1 , pp. $1-8,1990$.

4. T. Endo and L. O. Chua, "Chaos from phase-locked loops," IEEE Transactions on Circuits and Systems, vol. 35, no. 8, pp. 987-1003, August 1988.

5. T. Endo, L. O. Chua, and T. Narita, "Chaos from phaselocked loops part II: High-dissipation case," IEEE Transactions on Circuits and Systems, vol. 35, no. 2, pp. 255-263, February 1989.

6. V. N. Savov, Z. D. Georgiev, and T. G. Todorov, "Analysis of oscillations in quasi-conservative strongly nonlinear oscillator systems," IEEE Transactions on Circuits and SystemsI: Fundamental Theory and Applications, vol. 50, no. 12, pp. 1585-1588, December 2003.

7. V. N. Savov, Z. D. Georgiev, and T. G. Todorov, "Analysis and synthesis of perturbed Duffing oscillators," International Journal of Circuit Theory and Applications, vol. 34, pp. 281306, 2006.

8. M. Bonnin, "Harmonic balance, Melnikov method and nonlinear oscillators under resonant perturbation," International Journal of Circuit Theory and Applications, vol. 36, pp. 247274, 2008.

9. J. Guckenheimer and P. Holmes, Nonlinear Oscillations, Dynamical Systems and Bifurcations of Vector Fields, 5th ed. New York: Springer-Verlag, 1997.

10. J. Li, "Hilbert's 16th problem and bifurcations of planar polynomial vector fields," International Journal of Bifurcation and Chaos, vol. 13, no. 1, pp. 47-106, 2003.

11. C. Christopher and C. Li, Limit Cycles of Differential Equations, ser. Advanced Courses in Mathematics - CRM Barcelona. New York: Birkhäuser, 2006.

12. L. S. Pontryagin, "On dynamical systems close to Hamiltonian systems," Zh. Exp. \& Theor. Phys., vol. 4, pp. 234-238, 1934.

13. F. Dumortier and C. Li, "Perturbation from an elliptic hamiltonian of degree fouró-III global centre," Journal of differential equations, vol. 188, pp. 473-511, 2003.

14. G. S. Petrov, "On the nonoscillation of elliptic integrals," Functional Analysis and Its Applications,, vol. 31, no. 4, pp. 262-265, 1997.

15. Y. Zhao, "Zeros of Abelian integrals for the reversible codimension four quadratic centers $Q_{3}^{r} \bigcap Q_{4}^{*}$," Israel Journal of Mathematics, vol. 136, pp. 125-143, 2003.

16. M. Abramowitz and I. A. Stegun, Handbook of Mathematical Functions. Washington, DC: National Bureau of Standards, 1964. 